\title{
COMUNICAÇÃO EFETIVA NO TRABALHO EM EQUIPE EM SAÚDE: DESAFIO PARA A SEGURANÇA DO PACIENTE*
}

Jane Walkiria da Silva Nogueira ${ }^{1}$, Maria Cristina Soares Rodrigues²

Enfermeira. Mestranda do Programa de Pós-Graduação em Enfermagem da Universidade de Brasília. Brasília, DF, Brasil. ${ }^{2}$ Enfermeira, Farmacêutica. Pós-doutora em Ciências da Saúde. Docente do Departamento e de Enfermagem da Universidade de Brasília. Brasília, DF, Brasil.

RESUMO: Ensaio reflexivo que versa sobre a comunicação efetiva na perspectiva do trabalho da equipe interdisciplinar para a qualidade dos cuidados em saúde e a segurança do paciente. Na análise do objeto em tela, identificou-se que programas de treinamentos de habilidades de comunicação, simulações práticas e maneiras padronizadas para apresentar informações do paciente constituem formas efetivas para transpor barreiras à comunicação ineficaz. A adoção de estratégias para melhoria da comunicação da equipe representa desafio que requer mudança na cultura de segurança do paciente nas organizações de saúde.

DESCRITORES: Comunicação; Equipe de assistência ao paciente; Segurança do paciente.

\section{EFFECTIVE COMMUNICATION IN TEAMWORK IN HEALTH: A CHALLENGE FOR PATIENT SAFETY}

ABSTRACT: This reflexive essay discusses effective
communication from the perspective of multi-professional
teamwork for quality of healthcare and patient safety. In the
analysis of the object in question, itwas identified that training
programs for communication skills, practical simulations
and standardized ways for presenting information on the
patient constitute effective ways for overcoming barriers to
efficacious communication. The adoption of strategies for
improving the team's communication represents a challenge
which requires change in the culture of patient safety in the
health organizations. DESCRIPTORS: Communication; Patient care team; Patient safety.

\section{COMUNICACIÓN EFECTIVA EN EL TRABAJO EN EQUIPO EN SALUD: DESAFÍO PARA LA SEGURIDAD DEL PACIENTE}

RESUMEN: Ensayo que reflexiona sobre la comunicación efectiva en la perspectiva del trabajo del equipo interdisciplinario acerca de la cualidad de los cuidados en salud y la seguridad del paciente. En el análisis del objeto en pantalla, se identificó que programas de entrenamientos de habilidades de comunicación, simulaciones prácticas y modos estandarizados de presentar informaciones del paciente constituyen formas efectivas para superar las barreras de la comunicación ineficaz. El uso de estrategias para mejorar la comunicación del equipo representa desafío que pide cambio de cultura de seguridad del paciente en las organizaciones de salud.

DESCRIPTORES: Comunicación; Equipo de asistencia al paciente; Seguridad del paciente.

*Artigo originado da disciplina Segurança do Paciente, do Programa de Pós-Graduação em Enfermagem da Universidade de Brasília, 2014. 


\section{INTRODUÇÃO}

A cultura da segurança do paciente constitui um dos grandes desafios dos cuidados de saúde do século XXI. O reconhecimento da ocorrência de erros ou acidentes adversos com consequências danosas para pacientes e instituições de saúde levou a Organização Mundial da Saúde a nomear comissões centradas na identificação de situações de risco e na elaboração de soluções que possam servir de recurso para a prevenção dessas situações. Oresultado dos trabalhos das comissões tornou evidente a importância da comunicação e do trabalho da equipe interdisciplinar de saúde como determinante da qualidade e da segurança na prestação de cuidados aos indivíduos ${ }^{(1)}$.

Estudos apontam que falhas no trabalho em equipe e na comunicação entre os profissionais de saúde tem sido um dos principais fatores que contribuem para os erros médicos, eventos adversos (EAs) e, consequentemente, diminuição da qualidade dos cuidados ${ }^{(2-4)}$. Por conseguinte, a maneira como ocorre a comunicação entre os profissionais tem sidoapontada porpesquisadores como fundamental para um cuidado de saúde seguro $^{(5-6)}$.

Alguns fatores têm sido considerados cruciais para o desenvolvimento da comunicação efetiva entre os membros da equipe de assistência à saúde, tais como: contato dos olhos, escuta ativa, confirmação da compreensão da mensagem, liderança clara, envolvimento de todos os membros da equipe, discussões saudáveis de informações pertinentes, consciência situacional - esta se refere à compreensão do ambiente atual e à capacidade de antecipar com precisão problemas futuros ${ }^{(7)}$.

Neste sentido, tem-se presenciado na prática diária dificuldades dos profissionais de saúde em atuar em situações repentinas, inesperadas e que exigem atuação rápida dos membros da equipe, como por exemplo, na parada cardiorrespiratória, devido à falta de compreensão da atuação de cada cuidador neste tipo de atendimento. Tal realidade tem comprometido a qualidade e a segurança da assistência prestada, principalmente a pacientes de alto risco.

Considerando a relevância da temática em foco no campo da saúde e enfermagem contemporânea, associado à vivência prática das autoras em situações que são causadas por falhas no trabalho em equipe e na comunicação entre os profissionais da saúde, traçou-se como objetivo apresentar um ensaio reflexivo que versa sobre comunicação efetiva na perspectiva do trabalho da equipe interdisciplinar, para a qualidade dos cuidados em saúde e a segurança do paciente. Para tanto, realizou-se leitura analítica de artigos científicos, além de discussões realizadas em disciplina de pós-graduação stricto sensu em Enfermagem, com a triangulação dos temas comunicação, trabalho da equipe de saúde e segurança do paciente.

\section{COMUNICACÃO NO TRABALHO DA EQUIPE INTERDISCIPLINAR EM SAÚDE}

Pesquisas mostram que os profissionais prestadores decuidados de saúdetêm dificuldades de manter uma comunicação que favoreça o trabalho em equipe e, consequentemente, a segurança do paciente. Diferenças hierárquicas, poder e conflitos no contexto do trabalho no campo da saúde têm influenciado diretamente no modo como a comunicação se estabelece, fazendo com que as categorias profissionais atuem em paralelo, em detrimento do trabalho em equipe ${ }^{(2,5)}$.

Entre os principais desafios encontrados para a comunicação efetiva no trabalho em equipe da saúde, tem-se: a diversidade na formação dos profissionais, em que o treinamento para comunicação pode diferir entre os indivíduos; a tendência de uma mesma categoria profissional se comunicar mais uns com os outros; o efeito da hierarquia, geralmente com o médico ocupando posição de maior autoridade, situação que pode inibir os demais membros da equipe interdisciplinar ${ }^{(8)}$. Ademais, a literatura tem enfatizado a rigidez da hierarquia, que não permite criar um canal de comunicação efetiva com os diferentes níveis hierárquicos, pois não proporciona $\mathrm{o}$ compartilhamento das necessidades e os erros não são expostos

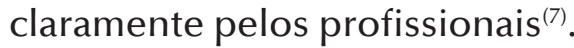

Sobre o tema em análise reflexiva, pesquisas mostram que é primordial o desenvolvimento de programas estruturados de treinamento de habilidades de comunicação, dos profissionais envolvidos diretamente (médicos, enfermeiros, técnicos de enfermagem, fisioterapeutas, entre outros) e indiretamente (gestores, agentes administrativos, entre outros) com o cuidado de saúde do paciente ${ }^{(7)}$. Tais programas têm proporcionado melhoras no desempenho e na comunicação entre os membros da equipe interdisciplinar ${ }^{(5-7)}$.

Em uma investigação conduzida com 
voluntários de um hospital norte-americano, realizou-se treinamento de habilidades de comunicação com obstetras e anestesistas. Os pesquisadores detectaram melhora significativa na integração e colaboração entre os médicos das especialidades, tornando $\mathrm{O}$ ambiente mais favorável para a comunicação adequada, necessária inclusive para a segurança do paciente $^{(9)}$.

O silêncio organizacional e a dificuldade de os profissionais se expressarem frente aos seus colegas de trabalho também tem sido considerado uma barreira para a comunicação e o trabalho em equipe. Um estudo investigou as preocupações que surgem entre médicos, enfermeiras e parteiras durante o processo de trabalho de parto e explorou como elas eram expressas. Identificou-se que, estes, muitas vezes, deixam de compartilhar problemas relacionados ao desempenho profissional, as lacunas do seu conhecimento, problemas de relacionamentos e a ocorrência de EAs. Apesar de reconhecerem que tal atitude coloca em risco a segurança do paciente, preferiram ficar em silêncio, sair de sua posição atual ou ser transferido do setor. Demonstrou-se assim que, quando as pessoas não expressam suas preocupações, os problemas que observam permanecem, uma norma de silêncio é reforçada e os membros da organização tornamse menos comprometidos ${ }^{(5)}$.

Portanto, a força do trabalho em equipe significa trabalhar com diferentes pessoas, com diferentes habilidades e conhecimentos para a gestão de um problema em comum e, desta forma, para que as equipes trabalhem o seu melhor, todos os membros devem compreender as habilidades e potencialidades de cada um dos seus colegas ${ }^{(7)}$.

Outro problema relacionado ao comprometimento de um processo de comunicação eficaz entre os profissionais tem sido a comunicação ocorrida nas passagens de turno (handover). Entre os problemas encontrados relacionados a esta prática, tem-se a omissão de dados importantes, falta de precisão ou consistência da informação, interrupções e ruídos frequentes que inviabilizam a clareza da mensagem a ser transmitida ${ }^{(1)}$. Número inadequado de profissionais também tem sido apontado como uma barreira para a comunicação eficaz, pois a fadiga pode provocar distrações e, consequentemente, falhas no processo de comunicação, como por exemplo, informações relevantes deixam de ser transmitidas ${ }^{(10)}$.
Pelo exposto, cabe a reflexão do necessário empenho das organizações de saúde e seus gestores com situações cotidianas, para conhecer dificuldades e desafios que o prestador do cuidado direto enfrenta diariamente, a fim de criar canal efetivo de comunicação com os níveis hierárquicos e, assim, permitir a construção da confiança entre todos os envolvidos. Como consequência, elos de confiança são firmados, as necessidades e os erros são expostos mais claramente pelos profissionais e a instituição consegue intervir nos processos de trabalho e na formação permanente, capacitando os profissionais para garantir uma cultura de segurança e uma assistência mais segura ${ }^{(11)}$.

\section{ESTRATÉGIAS PARA MELHORAR A COMUNICAÇÃO NAS ORGANIZAÇÕES DE SAÚDE}

\section{Trabalho em equipe e simulações multidisciplinares}

Estudos têm demonstrado que o trabalho em equipe resulta em maior produtividade, melhoria na comunicação e tomada de decisões. Além disso, proporciona aos profissionais melhora da autoestima, bem-estar psicológico e apoio social ${ }^{(5)}$. Nesta perspectiva, nos últimos anos, intervenções tem sido desenvolvidas, implementadas e avaliadas para melhorar o trabalho em equipe e a comunicação. Muitos gestores têm se apoiado em técnicas de treinamento em equipe baseada em simulações multidisciplinares, com ênfase em temas como liderança, consciência situacional, apoio e confiança mútua, comunicação e o papel de cada membro na equipe. Tais técnicas tem sido desenvolvidas para minimizar ou detectar erros, aperfeiçoar habilidades de trabalho em equipe, treinar/ensaiar procedimentos complicados e identificar lacunas de conhecimento dos profissionais relacionados à sua área de atuação profissional $^{(2-3,6)}$.

Atualmente, esse tipo de formação está se tornando amplamente aceita, e publicações científicas estão começando a demonstrar o seu papel para salvar vidas e melhorar resultados, principalmente em áreas como salas de parto, cirurgias, medicina de emergência, anestesia e medicina intensiva, que podem apresentar condições repentinas, inesperadas e raras, pois exigem resposta coordenada e rápida pela equipe interdisciplinar ${ }^{(3,10)}$. Portanto, a simulação pode ser usada para permitir que os profissionais pratiquem conceitos que aprenderam em um ambiente seguro, assim como tem o potencial para ajudar tanto na instrução inicial quanto no reforço do conceito. Além disso, se for feita in 
situ, é útil para avaliar o trabalho em equipe e a comunicação, como também aborda lacunas de conhecimento, resolução de conflitos, compartilha lições apreendidas e, ainda, identifica problemas reais relacionados à segurança dos pacientes $^{(3,10)}$.

\section{Autocorreção e protocolos de comunicação estruturados}

A literatura tem indicado ferramentas também consideradas cruciais para o trabalho em equipe e a comunicação, como: autocorreção, considerada fundamental para evitar erros e melhorar a qualidade; reuniões de equipes, fundamentais para evitar lapsos na transmissão formal de informações a todos os membros da equipe, promover espírito de equipe ${ }^{(1)}$; modelo/mapa mental compartilhado, ou seja, compreensão mútua dos problemas, metas e estratégias relacionadas com a situação em que se encontram ${ }^{(8)}$. Associados a essas iniciativas temse os protocolos de comunicação estruturados, como briefing (antes) e debriefings (depois) da execução de determinados procedimentos, como por exemplo, em cirurgias e partos. Antes dos procedimentos são discutidos vários assuntos que podem ser geradores de problemas, tais como comorbidades, falta de pessoal, cansaço, dificuldades, técnicas esperadas, entre outros, e após são discutidos os problemas identificados e a forma como a equipe lidou com eles ${ }^{(1-2,7)}$.

\section{Padronização de informações sobre o paciente}

Outra estratégia que tem sido abordada é a inclusãodemaneiraspadronizadasparaapresentar a informação do paciente, denominada SBAR (Situação; Background - história prévia; Avaliação; Recomendação). Essa tática fornece estrutura para a comunicação entre os profissionais a respeito da situação e da condição do paciente, e permite que os profissionais antecipem os próximos passos e alterem o seu modelo mental, se necessário ${ }^{(6-7)}$. Outras formas com a mesma finalidade incluem a verbalização da informação sobre o que é importante para toda equipe, especialmente em situações de emergência, e a repetição de volta à ordem e confirmar a informação recebida, a fim de evitar erros, por exemplo, da dose e via de medicações ${ }^{(7,10)}$.

\section{CONSIDERAÇÕES FINAIS}

Atualmente, ter qualidade e resultados positivos no setor saúde é exigência na gestão de processos de trabalhos coletivos. O trabalho em equipe em saúde é complexo e é considerado impulsionador de transformações, sendo a comunicação efetiva ponto-chave. Para uma cultura organizacional positiva, esse aspecto representa constante desafio, e requer avaliação permanente, em que pesem valores, hábitos, crenças, normas e experiências vivenciadas e compartilhadas pelos gestores e profissionais das organizações.

Vivências prévias no cotidiano profissional, assim como a literatura, têm indicado que a comunicação ineficaz repercute em cuidado inseguro, sendo um fator contributivo para desfechos desfavoráveis. Ao reflexionar sobre comunicação no trabalho de equipes interdisciplinares em saúde, a partir da produção científica utilizada neste ensaio, evidenciou-se que programas de treinamentos de habilidades de comunicação, simulações práticas e maneiras padronizadas para apresentar informações do paciente constituem formas efetivas para transpor barreiras à comunicação ineficaz.

Assim, uma cultura organizacional com missão embasada em objetivos e estratégias com efetivas configurações de comunicação, refletirá positivamente nos processos assistenciais e, consequentemente, na qualidade dos serviços e segurança do paciente.

\section{REFERÊNCIAS}

1. Santos MC, Grilo A, Andrade G, Guimarães T, Gomes A. Comunicação em saúde e a segurança do doente: problemas e desafios. Rev. Port. Sau Pu. [Internet] 2010; (10):47-57 [acesso em 10 nov 2014]. Disponível: https://www.ensp.unl.pt/dispositivos-de-apoio/cdi/ cdi/sector-de-publicacoes/revista/2010/pdf/volumetematico-seguranca-do-doente/6-Comunicacao $\% 20$ em $\% 20$ saude $\% 20$ e $\% 20$ a $\% 20$ seguranca $\% 20$ do $\% 20$ doente.pdf

2. Bagnasco A, Tubino B, Piccotti E, Rosa F, Aleo G, Pietro PD, et al. Identifying and correcting communication failure among health professional working in the Emergency Department. Int Emerg Nurs. 2013; 21(3):168-72.

3. Daniels K, Auguste T. Moving forward in patient safety: multidisciplinary team training. Semin Perinatol. 2013; 37(3):146-50.

4. Martins CCF, Santos VEP, Pereira MS, Santos NP. The nursing team's interpersonal relationships v. stress: limitations for practice. Cogitare enferm. [Internet] 2014; 19(2): 287-93 [acesso em 10 fev 2015]. Disponível: http://ojs.c3sl.ufpr.br/ojs/index.php/cogitare/article/ viewFile/36985/22757

5. Maxfield DG, Lyndon A, Kennedy HP, Keeffe DO, 
Zlatnik MG. Confronting safety gaps across labor and delivery teams. Am J Obstet Gynecol. 2013; 209(5):4028.

6. Lee P, Allen K, Daly M. A "Communication and Patient Safety" training programme for all healthcare staff: can it make a difference? BMJ Qual Saf. 2012; 21(1): 84-8.

7. Johnson HL, Kimsey D. Patient safety: break the silence. AORN J. 2012; 95(5): 591-601.

8. Rowlands S, Callen J. A qualitative analysis of communication between members of a hospitalbasead multidisciplinar lung cancer team. Eur J Cancer Care. 2013; 22(1):20-31.

9. Kirschbaum KA, Rask JP, Brennan M, Phelan S, Fortner SA. Improved climate, culture, and communication through multidisciplinary training and instruction. Am J Obstet Gynecol. 2012; 207(3):200.e1-7.

10. Deering S, Johnston LC, Colacchio K. Multidisciplinary teamwork and communication training. Semin Perinatol. 2011; 35(2):89-96.

11. Paese F, Dal Sasso GTM. Cultura da segurança do paciente na atenção primária à saúde. Texto Contexto Enferm. 2013; 22(2):302-10. 